\title{
THE TWENTY-NINTH ANNUAL MEETING OF THE SOCIETY
}

The twenty-ninth annual meeting of the Society was held at Harvard University on Wednesday and Thursday, December 27-28, 1922, during the period of the Convocation week meetings of the American Association for the Advancement of Science and immediately preceding the meeting of the Mathematical Association of America. Dormitory accommodations were furnished by Harvard University and Radcliffe College, and Phillips Brooks House was used as a social center for the mathematical group. Not only was there a record attendance at the meeting, but members found it stimulating and pleasant to come into contact with scientists working in fields related to mathematics. At the close of the sessions it was voted to express the thanks of the Society to Harvard University for its hospitality and to the departments of mathematics at both Harvard and the Massachusetts Institute of Technology for the admirable way in which arrangements for the meetings were carried out.

Members of the Society were invited to hear the address of Professor E. H. Moore, retiring president of the American Association for the Advancement of Science, on What is a number system?, delivered Tuesday evening at the Massachusetts Institute of Technology. Joint sessions were held on Wednesday afternoon with Section A of the American Association for the Advancement of Science, at the Massachusetts Institute of Technology, and on Thursday afternoon with the Mathematical Association of America and Section A at Harvard University. An enjoyable occasion was the joint dinner of the three organizations held at the Massachusetts Institute of Technology on Thursday evening.

The attendance included the following 148 members of the Society:

Alexander, E. S. Allen, Archibald, Atchison, Bailey, Beatley, W. J. Berry, Bill, Birkhoff, Bliss, Borden, Bowler, B. H. Brown, E. P. Brown, Bryan, M. Buchanan, W. G. Bullard, R. W. Burgess, Burkett, Burwell, 
Cairns, Cajori, B. H. Camp, Carr, B. E. Carter, Clawson, Coates, Coolidge, Copeland, Crum, Currier, Denton, Dimick, Dodd, Eisenhart, L. C. Emmons, Esty, G. C. Evans, G. W. Evans, Falconer, Floyd Field, Fields, C. H. Forsyth, Fort, Philip Franklin, Frizell, Garabedian, Gerst, Gilman, Gleason, Glenn, Glover, Gould, M. C. Graustein, W. C. Graustein, Gummer, Hammond, J. G. Hardy, Haskins, Hazlett, Hicks, Hille, Hodgkins, Huntington, Hurwitz, Ingraham, Kazarinoff, Kellogg, Kennelly, W. D. Lambert, Larew, Leib, Leyzerah, Lipka, McGavock, MacDuffee, MacPherson, H. P. Manning, Marriott, G. A. Miller, Molina, C. L. E. Moore, C. N. Moore, E. H. Moore, R. K. Morley, H. C. M. Morse, F. R. Moulton, F. H. Murray, Musselman, Newkirk, Olds, Osgood, Packer, Pattillo, Pell, Perkins, Phillips, Pitcher, Ransom, Ranum, L. H. Rice, R. G. D. Richardsons, H. L. Rietz, E. D. Roe, Roever, Ruger, Rutledge, Safford, Seely, Sharpe, Shaub, Shewhart, Silverman, W. G. Simon, Sinclair, Slaught, Slobin, C. E. Smith, D. E. Smith, S. E. Smith, M. J. Sperry, Stetson, Swartzel, J. S. Taylor, J. M. Thomas, Tracey, Tyler, Van Vleck, Veblen, Vivian, Waddell, J. L. Walsh, Webster, Wedderburn, Weisner, M. E. Wells, H. S. White, D. E. Whitford, Whittemore, Wiener, C. E. Wilder, F. B. Williams, Willis, R. G. Wood, F. S. Woods, J. M. Young, J. W. Young, Zeldin.

President Bliss presided at the session Wednesday morning, relieved by Professor Veblen. President Veblen presided at the joint session on Wednesday afternoon, Professor Osgood on Thursday morning, and Professor G. A. Miller, VicePresident of Section A, at the joint session on Thursday afternoon.

At the meeting of the Council, the following twenty-two persons were elected to membership in the Society:

Professor Ralph Beatley, Harvard Graduate School of Education;

Mrs. Ethelwynn Rice Beckwith, Harvard University;

Rev. Dr. Martin Brennan, St. Louis, Mo.;

Professor James Bruce Coleman, University of South Carolina;

Professor Marion Alonzo Eason, United States Naval Academy;

Mr. Hervey Crandall Hicks, Brown University;

Mr. William Charles Janes, Kansas State Agricultural College;

Mr. Carl Otto Lampland, Lowell Observatory;

Mr. Frank Solimena Langellotti, New York, N. Y.;

Miss Martha Peirce McGavock, Wellesley College;

Mr. Albert Eugene Meder, Columbia University;

Dr. Winifred Edgerton Merrill, Mamaroneck, N. Y.;

Dr. Harvey Pierson Pettit, University of Illinois;

Mr. Pincus Schub, Hebrew School of Newark;

Professor Charles Cutler Spooner, Northern State Normal School, Marquette, Mich.;

Mr. Charles Aynes Stanwick, Western Electric Company; 
Professor Tsurusaburo Takasu, Tôhoku Imperial University;

Dr. Louis Ten Fyck Thompson, Kalamazoo College;

Dr. Elihu Thomson, General Electric Company;

Professor Edward Lee Thorndike, Teachers College, Columbia University; Miss Virginia Watts, New Orleans, La.;

Mr. Frederick W. Weston, Wynnewood, Pa.

Twenty-five applications for membership were received. The Secretary announced that the following members of the London Mathematical Society had entered the American Mathematical Society in consequence of the reciprocity agreement recently arranged between the two Societies:

Professor Mathew J. Conran, University College, Cork;

Mr. Krishna Prasad Dè, University College, Rangoon;

Professor Arthur Lee Dixon, Magdalen College, Oxford;

Principal John Leigh Smeathman Hatton, East London College, University of London;

Professor Harold Hilton, Bedford College, University of London;

Sir Joseph Larmor, St. John's College, Cambridge;

Professor George Henry Livens, University College, Cardiff;

Professor William Proctor Milne, University of Leeds;

Professor Archibald Read Richardson, University College, Swansea;

C. M. Ross, King's College, Cambridge;

Mr. Reginald Owen Street, University of Liverpool;

Mr. Bertram Martin Wilson, University of Liverpool.

The Council announced that the invitation of Vassar College to hold the summer meeting of 1923 at that institution had been accepted and that the annual meeting would be held in New York City.

The committee on membership made a final report through its chairman, President Bliss. Since October 1921 the committee by enlisting the cooperation of several members of the Society has obtained some 228 new members, 33 new subscriptions to the Transactions and $\$ 372.00$ in gifts from members to the regular funds. It recommended that in view of the still critical state of the finances of the Society a new committee on membership be appointed. In discharging the committee, the Council thanked the chairman for the splendid service rendered to the Society. A new committee on membership was appointed consisting of Professors Abraham Cohen, W. H. Roever, and C. E. Smith (permanent secretary). 
Professor G. A. Bliss was elected a member of the International Mathematical Union to fill the vacancy caused by Professor Veblen's succession to the presidency of the Society. Professors C. N. Moore and R. G. D. Richardson were appointed to represent the Society on the Council of the American Association for the Advancement of Science for the year 1923.

The Council authorized the appointment by the President of standing committees on endowment, and on policy and budget.

The total membership of the Society is now 1127, including 82 life members. The total attendance of members at all meetings, including sectional meetings, during the past year was 538; the number of papers read was 228 . The number of members attending at least one meeting was 377 . At the annual election 246 votes were cast.

The reports of the Treasurer and the Auditors (Mr. S. A. Joffe and Professor W. J. Berry) were received, showing a balance $\$ 13,232.36$, exclusive of special funds; of this $\$ 5782.85$ is reserved to secure the life memberships. It was explained that the large balance on hand is due to the fact that the Transactions of the Society is eighteen months late in appearing. Sales of the Society's publications during the year amounted to $\$ 3,298.43$.

The Library now contains 6,203 volumes, exclusive of members' dissertations.

At the annual election, which closed on Wednesday morning, the following officers and other members of the Council were chosen:

President, Professor Oswald Veblen.

Vice-Presidents, Professor R. L. Moone, Professor H. W. TyLer.

Secretary, Professor R. G. D. Richardson.

Treasurer, Professor W. B. Fite.

Librarian, Professor R. C. ARchibald.

Committee of Publication

Professors E. R. Hedrick, W. A. Hurwitz, J. W. Young. 
Members of the Council to serve until December, 192.5

Professor E. T. Bell, Professor J. C. Fields, Professor W. H. Bussey, Professor Arthur Ranum.

The amendment to the constitution designating as permanent member of the Council any ex-Secretary who had served ten years was carried.

The joint session on Wednesday afternoon was devoted to a symposium on Space and Time and was attended by about three hundred scientists. The following papers were read:

I. The logic of space and time, by Professor G. D. Birkhoff.

II. The physical meaning of space and time, by Professor P. W. Bridgman.

III. The astronomical measures of space and time, by Professor Harlow Shapley.

The program of the joint session on Thursday afternoon was as follows:

I. The reduction of singularities of plane curves by birational transformation, by Professor G. A. Bliss, retiring President of the Society. This paper will appear in full in the April number of this BuLLETIN.

II. The grafting of the theory of limits on the calculus of Leibniz, by Professor Florian Cajori. (Address delivered at the request of the Mathematical Association of America.)

III. Geometry and physics, by Professor Oswald Veblen, retiring Vice-President of Section A.

Titles and abstracts of the papers read at the regular sessions of the Society follow below. Dr. Bray's paper was read by Professor Evans. The papers of Professors Graustein, C. L. E. Moore, Craig, Cajori, Bell, R. L. Moore, and Camp, Dr. Post, Professor Schwatt, Mr. Poritsky, Professors Hitchcock and Cowley, Mr. Murray, Professor Alexander, and Mr. Raynor, and the second papers of Dr. Walsh, Dr. B. H. Brown, Dr. Wiener, Dr. Franklin, Professor Coolidge, Professor Miller, and Dr. MacDuffee were read by title. Mr. Brinkmann and Mr. Linfield were introduced by Professor Birkhoff and Professor Hitchcock by Professor C. L. E. Moore. 
1. Dr. J. L. Walsh: On the location of the roots of polynomials.

It is the purpose of this paper to prove the following theorem: Let circles $C_{1}, C_{2}, \cdots, C_{k}$, whose centers are the points $\alpha_{1}$, $\alpha_{2}, \cdots, \alpha_{k}$, be the loci of $n_{1}, n_{2}, \cdots, n_{k}$ roots of a polynomial $f(z)$ which has no other roots, where the circles $C_{i}$ are all equal and their centers $\alpha_{i}$ all lie on a line parallel to the axis of reals. If these circles are sufficiently small, and if the polynomial

$$
\begin{array}{r}
a_{0} z^{n}+n a_{1} z^{n-1}+n(n-1) a_{2} z^{n-2}+\cdots+n(n-1) \cdots 2 \cdot 1 a_{n}, \\
\left(n=n_{1}+n_{2}+\cdots+n_{k}\right),
\end{array}
$$

has only real roots, then the locus of the roots of the polynomial $F(z)=a_{0} f(z)+a_{1} f^{\prime}(z)+a_{2} f^{\prime \prime}(z)+\cdots+a_{n} f^{(n)}(z)$ consists of the circles $C_{j}^{\prime}$ that are equal to the circles $C_{i}$ and whose centers are the roots of $F(z)$ when the roots of $f(z)$ are the points $\alpha_{1}, \alpha_{2}, \cdots, \alpha_{k}$ of multiplicities $n_{1}, n_{2}, \cdots, n_{k}$ respectively. Any circle $C_{j}^{\prime}$ which has no point in common with any of the other circles $C_{m}{ }^{\prime}$ contains a number of roots of $F(z)$ equal to the multiplicity of its center as a root of $F(z)$ when the roots of $f(z)$ are the points $\alpha_{i}$.

2. Dr. J. L. Walsh: A generalization of normal congruences of circles.

This paper appeared in the December Bulletin.

3. Dr. Eleanor P. Brown: Expansion theorems for a certain homogeneous integral equation.

This paper deals with a Fredholm linear homogeneous integral equation $u(x)=-\rho^{2} \mathcal{S}_{\alpha}^{\beta} K(x, \xi) u(\xi) d \xi$, whose kernel is such that, though continuous and possessing continuous derivatives on either side of the line $x=\xi$, in the $x$, $\xi$ plane, it has certain "finite jumps" in its derivatives as the point $(x, \xi)$ crosses this line. Provided certain orthogonality relations are not satisfied, the equation can be reduced to a differential equation of Sturm-Liouville type with the addition of certain integral terms, along with a pair of boundary conditions. These integral terms have little effect on the solutions of the equation, and it is shown that the characteristic numbers and functions behave essentially like those of the corresponding Sturm-Liouville system. The fundamental theorem is proved that if we set up the formal Fourier series, with respect to each of the two above-mentioned systems, of any function $\Phi(x)$ which, in absolute value, is integrable, then the difference 
(taken termwise) between these two series converges absolutely and uniformly to zero.

4. Dr. B. H. Brown: The equilong transformations of euclidean space.

The equations of the equilong transformations of euclidean space, in Bonnet oriented tangential coordinates, are

Direct: $\quad U=U(u), \quad V=V(v), \quad W=\sqrt{U^{\prime} V^{\prime}} w+F(u, v)$; Indirect: $U=U(v), \quad V=V(u), W=\sqrt{U^{\prime} V^{\prime}} w+F(u, v)$.

The forms given by Blaschke (ARCHIv, 1910), and all subsequent references in the literature contain $a \pm$ sign under the radicals, which is incorrect.

The main portion of this paper deals with the invariance of differential expressions and equations under these, or subgroups of these transformations. Applications are made to one-sided surfaces, to minimal surfaces and their associated surfaces, and to a large class of surfaces defined by second order linear partial differential equations, including the surfaces of Appell, of Goursat, and of Bonnet. The following theorem is proved: Any oriented non-developable surface may, by each of two and only two distinct, direct, equilong transformations, be transformed into any other oriented nondevelopable surface, and that with an arbitrary analytic directly conformal mapping of their spherical representations. 'This theorem was suggested by Study, but he stated, incorrectly, that there was one and only one such transformation.

5. Dr. B. H. Brown: Contact transformations linear in $x, y$, and $z$; applications to equilong transformations.

A transformation of Lie planar elements, in which $X, Y$, and $Z$ are linear in $x, y$, and $z$, carries planes into planes if and only if all the three-rowed minors of a certain ten-by-three matrix vanish. Necessary and sufficient conditions for equilong transformations, a subgroup of the preceding group, follow easily. For a particular subgroup of the equilong group, these conditions reduce to two partial differential equations which are necessary and sufficient conditions that the parameters $u$ and $v$ of a general surface $x=x(u, v)$ have the geometric significance of $p$ and $q$. Certain rational solutions of these equations furnish the parametric equations of well known one-sided surfaces. The transformation of Bonnet is shown to be equilong. 
6. Professor W. C. Graustein: On the determination of a surface by its principal normal curvatures and spherical representation.

It is shown in this paper that a non-developable surface is, in general, uniquely determined, to within its position in space, by the linear element of its spherical representation and its principal normal curvatures, and that the exceptional cases, for which definitive conditions are given, lead either to two or to a single infinity of non-congruent surfaces.

7. Professor W. C. Graustein and Mr. B. O. Koopman: $A$ necessary and sufficient condition that a map of two surfaces be isometric.

It is well known that in order that a map of two surfaces be isometric it is necessary that it be geodesic and that the total curvature at corresponding points be equal; it is also known that neither of these conditions is alone sufficient. This paper shows that these two conditions taken together are sufficient, except when the two surfaces are of constant total curvature.

8. Professor C. L. E. Moore: Deformation of surfaces in 4-space.

The author derives characteristic equations which must be satisfied if two surfaces correspond by orthogonality of elements, and shows the connection with infinitesimal deformation.

9. Professor Joseph Lipka: On conformal parallelism.

We consider two curved spaces $V_{n}$ and $V_{n}^{\prime}$, one of which is a conformal representation of the other. Two linear elements in $V_{n}$ are conformally parallel when their conformal representations in $V_{n}^{\prime}$ are parallel in the sense of Levi-Civita. The author proves a number of theorems on conformal parallelism in $V_{n}$ analogous to the theorems on parallelism in $V_{n}{ }^{\prime}$. A natural family of curves in $V_{n}$ plays the rôle of the geodesics in $V_{n}^{\prime}$. In particular, a study is made of the motion of a linear element by conformal parallelism around an infinitesimal cycle, and of the motion of a linear element both by parallelism and conformal parallelism along a curve.

10. Professor Joseph Lipka: On irreversible systems in dynamics.

An irreversible field of force is defined as one in which the Lagrangian or kinetic potential has the form

$$
L=L_{2}+L_{1}+L_{0}=\frac{1}{2} \sum_{i k} a_{i k} \frac{d x_{i}}{d t} \frac{d x_{k}}{d t}+\sum_{i} \phi_{i} \frac{d x_{i}}{d t}+U,
$$


where $t$ is the time, and $a_{i k}, \phi_{i}$, and $U$ are functions of the coordinates only. In this paper, the author proves the theorem that if in an irreversible field of force with a given constant of energy, particles are projected from all points of an arbitrary surface in a direction such that the vector sum of the velocity and the vector $\phi_{i}$ is normal to the surface, points which they reach with equal actions (action $=\mathcal{S}\left(2 L_{2}+L_{1}\right) d t$ ) lie on a surface also cutting the paths so that the vector sum of the velocity and the vector $\phi_{i}$ is normal to the surface. (If the vector $\phi_{i}$ vanishes, there is a reversible field, and the theorem reduces to the well known theorem of Thomson and Tait.) By means of Hamilton's canonical equations and the theory of contact transformations, this theorem and its converse are proved directly for curved spaces of any dimensionality.

11. Dr. J. S. Taylor: A 4-space representation of complex plane analytics.

Kwietniewski and K. Kommerell have set up a one-to-one correspondence between a certain family of surfaces in ordinary 4 -space $S_{4}$ and the curves (real and imaginary) of plane analytics. In the present paper the author investigates the problem of representing plane analytics as a whole in a modified 4 -space $\Sigma_{4}$, extending for use in the 4 -space the complex measures of distance and angle of complex plane analytics. The "rigid motions" of $S_{4}$ and of $\Sigma_{4}$ are discussed, both in relation to each other and in relation to the transformations effected on equations defining regular functions of a complex variable. Among other subgroups, the group of rotations in $S_{4}$ is obtained under which all surfaces representing regular functions remain surfaces representing regular functions. Incidentally, a family of 4 -space rotations is given that has apparently been overlooked. The author makes some applications to the theory of functions of a complex variable.

12. Dr. Norbert Wiener: Differential-space.

The author shows that procedure to the limit in questions relating to the Brownian movement gives rise to a space of infinitely many dimensions of a character fundamentally distinct from the familiar function-space discussed by such writers as Fréchet, Volterra, and Lévy, and employs the methods of Lévy in developing integration in this space.

He discusses the introduction of orthogonal coordinates in differential-space, and develops a complete set of normal and orthogonal functionals, related to the Hermite polynomials. 
13. Dr. Norbert Wiener: Note on the series $\Sigma( \pm 1 / n)$.

The author shows that with an arbitrary haphazard selection of signs for the terms of $\Sigma( \pm 1 / n)$, it is of zero probability that it diverge.

14. Professor J. L. Coolidge: On the existence of curves with assigned singularities.

This paper appeared in the December Bulletin.

15. Professor J. L. Coolidge: The Gaussian law of error for any number of variables.

In this paper the Gaussian law of error is developed for any number of variables, by a natural extension of the classical method, and the coefficients are determined in terms of individual mean errors and correlation coefficients.

16. Mr. H. W. Brinkmann: On conformal Riemann spaces.

The author proves the following theorem: An $n$-dimensional Riemann space conformal to euclidean space can be considered as the intersection of a null hypersphere and some other hypersurface in $(n+2)$-dimensional euclidean space. By means of this theorem Schouten's necessary and sufficient condition that a Riemann space be conform-euclidean is readily derived. The method of proof is entirely elementary and can be extended to prove a similar theorem for any two conformal Riemann spaces. Theorems concerning Riemann spaces containing certain sets of $n$-fold orthogonal systems of hypersurfaces are also obtained by this method.

17. Professor E. S. Allen: On certain power series with positive coefficients.

Each coefficient of a series of the type considered is a function of all preceding coefficients. Information is obtained concerning the interval of convergence, in some cases through the expression of the coefficients in closed form.

18. Dr. Philip Franklin: A qualitative definition of the trigonometric and hyperbolic functions.

This paper appeared in the February Bulletin.

19. Dr. Philip Franklin: Tensors of given types in Riemann space.

This paper begins by defining an arithmetic tensor in euclidean space as a tensor whose components are constant, independent of both the position of the point in question and 
the cartesian coordinates used, and proving some of its properties. These properties are then extended to tensors in Riemann space, the final theorem being: The most general tensor of given even order involving the $g_{i j}$ 's and their first and second derivatives, these last linearly, is a linear combination of tensors which may be formed from the curvature tensor $R_{f g h}^{e}$ and the fundamental quadratic tensor $g_{i j}$ by multiplication and contraction. There are no such tensors of odd order.

These theorems enable us to set up many of the equations used in physics by taking the most general tensor of the type wanted, and after determining the constants by special considerations equating it to zero. The paper has been accepted for publication by the Philosophical Magazine.

20. Mr. B. Z. Linfield: A contribution to particle geometry.

In this paper the varieties of a universe of particles are classified according to the nature of the subvariety which is embedded in a variety and which is in the neighborhood of an arbitrary element (particle) of this same variety. After considering the $n$-dimensional, normal varieties of the second order, $S_{n}^{2}$, the author concludes with the following theorem: There exists a regular map of regions whose boundaries are convex which is equivalent to an. arbitrary regular map.

21. Dr. H. E. Bray: Proof of a formula for an area.

This paper will appear in full in this Bulletin.

22. Professor C. F. Craig: On the Riemann zeta function.

In this note a functional relation for the Riemann zeta function is established and its relation to the well known formula given by Riemann is pointed out. It is believed that the relation is new.

23. Professor Florian Cajori: Mathematical signs of equality.

Recorde's sign $=$ of 1557 did not again appear in print until 1618, but after 1631 it gained a firm foothold in England. Certain Continental writers used $=$ in the seventeenth century in four different senses. Seven different signs of equality were proposed, but Descartes' $\infty$ was the only serious rival, which about 1680 threatened to displace $=$ on the Continent. The two inventors of the calculus, Leibniz and Newton, favored $=$ and apparently brought about its final victory.

24. Professor E. T. Bell: Extended class number relations.

A sum of the form $\Sigma X\left(n-\sigma^{2}\right)$ in which $X$ is any class number function and $\Sigma$ extends to certain of the integers $\sigma$ 
rendering $n-\sigma^{2}>0$ is here called principal of order $c$ and degree $\mu$ if each $\sigma / c$ is the $\mu$ th power of an integer. A relation involving a principal sum of order $c$ and degree $\mu$ is called extended of rank $\lambda$ and range $(A, B)$, where $A<B$, if the relation involves also the arbitrary even function $f$ having as arguments only integers whose $\lambda$ th powers lie between $A$ and $B$. By a simple new method a closely interconnected set of 29 such extended relations is found in which $c$ is an arbitrary constant integer $\neq 0, \mu$ is an arbitrary odd constant integer $>0, \lambda$ is an arbitrary even constant integer $>0$, and $A, B$ $(A<B)$ are arbitrary constants. Hitherto only instances of the cases $c=1,4$, or a prime, $\mu=1, \lambda=2, A=0, B=\infty$, $f(x)=1$ for all values of $x$ have appeared. For these special values the extensions degenerate to known relations of Kronecker, Liouville, and Humbert, or to others of similar types.

25. Professor R. L. Moore: Concerning the uniform convergence of certain sequences of equicontinuous arcs.

Suppose that (1) $A B$ and $C D$ are two parallel lines, (2) for every $n, A_{n}$ and $C_{n}$ are points lying on $A B$ and $C D$ respectively and $A_{n} C_{n}$ is a simple continuous arc which, except for its end points, lies between $A B$ and $C D$, (3) the sequence of arcs $A_{1} B_{1}, A_{2} B_{2}, \cdots$ is equicontinuous and has, as its limiting set, an arc $A C$ which lies, except for its end points, entirely between $A B$ and $C D$. Then there exists, for each $n$, a one-to-one continuous correspondence, $T_{n}$, between $A C$ and $A_{n} C_{n}$ such that the set of correspondences $T_{1}, T_{2}, T_{3}, \ldots$ is equicontinuous and such that, with respect to this set of correspondences, the sequence $A_{1} C_{1}, A_{2} C_{2}$ converges uniformly to $A C$, that is to say, it is true that for every positive number $\epsilon$ there exists a positive number $\delta_{\epsilon}$ such that if $n>\delta_{\epsilon}$ then, for every point $P$ of $A C$, the distance from $P$ to $T_{n}(P)$ is less than $\epsilon$.

It is shown that a sequence of $\operatorname{arcs} A_{1} C_{1}, A_{2} C_{2}, \ldots$ is not necessarily equicontinuous * even though there exist two equicontinuous sequences of functions $f_{1}(t), f_{2}(t), \cdots$ and $F_{1}(t), F_{2}(t)$, -.. such that, for every $n, A_{n} C_{n}$ is represented parametrically by the pair of equations $x=f_{n}(t)$ and $y=F_{n}(t)(0 \leqq t \leqq 1)$.

26. Professor R. W. Burgess: A non-linear partial regression equation.

In the theory of partial regression equations, it is assumed that each variable is a linear function of the others. This assumption implies, in the case of three variables, that the coefficient of regression of one variable on a second is constant

*Cf. Transactions of this Society, vol. 22 (1921), p. 42. 
no matter what values are assigned to the third variable. As in certain statistical problems this seems contrary to the essential nature of the relationship between the variables, the author suggests the use of the equation $x_{1}=a x_{2} x_{3}+b x_{2}$ $+c x_{3}+d$, and has determined the best values of the coefficients $a, b, c, d$, in the sense of the method of least squares. He has applied his method to a further analysis of $\mathrm{H}$. L. Moore's problem of predicting the yield of cotton from the May rainfall and June temperature, and finds that the new method results in an improvement in accuracy of prediction.

27. Professor B. H. Camp: On a short method of least squares.

It is known that, when the independent variables of a set of observational equations are equi-spaced, certain simplifications are available which greatly shorten the least square solution (cf. H. M. Roeser, Scientific Papers of the Bureau of Standards, No. 388). It is shown by the author that these may frequently be employed, with negligible error, when the independent variables are only approximately equi-spaced. Expressions for the error are derived which may be computed quickly, after the short solution has been obtained, and which also permit the computer to estimate roughly, in advance of his computation, the size of the error to be expected. This paper will appear in the Annals of Mathematics.

28. Professor Olive C. Hazlett: Finiteness theorems for formal modular covariants.

By the aid of a symbolic notation explained in a previous paper, the author proves that all formal modular covariants of a system of binary forms with respect to the Galois field $G F\left[p^{n}\right]$, of order $p^{n}$, are congruent, in the field, to polynomials in a finite number of such covariants.

29. Professor R. W. Marriott: Determination of all types of subgroups of the group of order $p^{4}$, where $p$ is a prime.

The exact number of every type of subgroup of order $p^{\alpha}$, $\alpha<4$, has been determined, in this paper, for every group of order $p^{4}$, and these subgroups expressed in terms of the generating operations of the group of order $p^{4}$. The following theorem was made use of: Let $G p^{m}$ be a group of order $p^{m}$ of a certain given type, and $G p^{a}$ a group of order $p^{a}$ of specified type, $\alpha<m$. Let $N$ be the number of ways operations can be selected from $G p^{m}$ which satisfy the defining relations of $G p^{a}$, but no relations independent of these. Then the number of subgroups of $G p^{m}$ of order $p^{a}$ and type $G p^{a}$ is equal to $N$ divided by the order of the group of isomorphisms of $G p^{a}$. 
The author takes for generating elements of $G p^{a}$ the most general operations in the group of order $p^{m}$, subjèct these operations to the defining relations of $G p^{a}$, and impresses on them the further condition that they shall obey no relations other than these defining relations of $G p^{a}$. The method leads always to a set of congruences involving the exponents of the generators, the number of solutions of which gives the value of $N$.

\section{Professor O. E. Glenn: The sieve of Eratosthenes.}

The distribution of primes in the infinite sequence of positive integers is the subject of many researches dating from the work of Eratosthenes (276 B. C.). Tables of figurate numbers defined in the author's paper presented at the Chicago meeting, April, 1922, contain primes distributed practically at random. The method there explained for finding the figurate numbers which are divisible by a given "prime $p$ is equivalent to a generalization to two dimensions of Eratosthenes' sieve. We prove that for extensive classes of plexuses the complete description of divisibility by $p$ may be obtained from a finite period parallelogram of residues recurrent to infinity within the plexus, and the method of the sieve gives general results. The developments of the paper connect with some ancient problems in numbers and the finished manuscript is to present extensive lists of primes from typical figurate number tables.

31. Professor G. A. Miller: New applications of a fundamental theorem of substitution groups.

The fundamental theorem considered in this paper may be stated as follows: If the cycle $a_{1} a_{2} \cdots a_{l}$ appears in at least one substitution of the group $G$ and if we count this cycle and jts conjugates under $G$ as many times as each of them appears in different substitutions of $G$, then the total number of letters in these cycles is equal to the order of $G$. From this theorem one can readily obtain the following useful theorem: The number of the different sets of conjugate cycles under any substitution group of degree $n$ is $n-k, k$ being the number of its systems of intransitivity. In particular, all the possible transitive groups of degree $n$ have in common the property that each of them contains the same number of sets of conjugate cycles. The author aims to exhibit the applications of these theorems by using them in a new determination of the substitution groups of degrees four and five, and in finding exponents to which numbers belong with respect to a modulus which is a power of a prime number. 
32. Professor G. A. Miller: New proof of the theorem that every prime power group contains invariant operators.

The author shows that his proof of Sylow's theorem* can readily be extended to include a proof of the fundamental theorem that every group of order $p^{m}$ ( $p$ a prime) contains at least one invariant operator of order $p$. To prove this extension it is convenient to let $p^{\beta}$ represent the highest power of $p$ which divides $n$ instead of the highest power of $p$ which does not exceed $n$, since in both cases the number of substitutions of degree $p^{\beta}$ and order $p$ in the symmetric group of degree $n$ is prime to $p$. The regular group $G$ whose order is divisible by $p^{\beta}$ but not by $p^{\beta+1}$ contains a subgroup of order $p^{\beta}$ whose constituents are regular, and the substitutions of the symmetric group of degree $n$ which are of degree $p^{\beta}$ and commutative with each substitution of this subgroup of order $p^{\beta}$ must be found in the conjoints of these regular constituents. As the number of these conjoints is prime to $p$, each of them contains a number of substitutions of order $p$ which is prime to $p$, and the subgroup of order $p^{\beta}$ contains a number of substitutions of order $p$ which is prime to $p$, and therefore an invariant operator of order $p$. It follows also the number of operators of order $p$ in a group whose order is divisible by $p$ is prime to $p$.

33. Dr. C. C. MacDuffee: Covariants and transformable systems.

This paper appeared in the January Bulletin.

34. Dr. C. C. MacDuffee: A theorem on covariants of forms with an application to linear algebras.

It is proved that for every value of $j$ the parameters $\alpha_{i j}$ of a linear homogeneous transformation replacing the system of forms $f_{k}$ by the system $f_{k}{ }^{\prime}$ are transformed by an arbitrary transformation cogrediently with the variables $x_{i}$. This theorem gives a means of calculating the rational integral covariant of the linear algebra in $n$ units (one of which is a principal unit) which vanishes identically when and only when the right-hand (left-hand) rank of the algebra is less than $n$.

35. Professor C. N. Moore: On the Cesàro summability of the double Fourier's series.

The main result of this paper is the theorem that the double Fourier's series corresponding to any function $f(x, y)$ that has a Lebesgue integral in the region $(-\pi \leqq x \leqq \pi,-\pi$

*AnNals of Mathematics, (2), vol. 16 (1915), p.169. 
$\leqq y \leqq \pi)$ will be summable $(C, \rho, \delta)$, where $\rho$ and $\delta$ are any two positive constants, throughout the above region, except for a set of points of measure zero. This is the extension to double Fourier's series of a theorem with regard to ordinary Fourier's series due to G. H. Hardy.

36. Professor L. L. Silverman: A certain type of regular transformation.

In the study of regular transformations of the form $y_{n}$ $=\sum_{k=1}^{n} a_{n k} x_{k}$, several authors have defined $a_{n n}$ by means of a function $f(x)$ satisfying certain conditions in the interval $(0,1)$, and have then defined the numbers $a_{n k}, k<n$, by the condition that the given transformation be permutable with the special transformation $y=(1 / n) \sum_{k=1}^{n} x_{k}$. These transformations, which we shall call of type $A$, include the definitions of Cesàro and Hölder for special choice of $f(x)$. The present author studies the transformations of type $B$, for which $a_{n n}=1$ $-f((n-1) / n), \quad a_{n k}=f(k / n)-f((k-1) / n), k<n$, where $f(x)$ satisfies the same conditions in the interval $(0,1)$ as in the case of transformations of type $A$. An objection to this new type of transformation is that for no choice of $f(x)$ does it represent any of the transformations of Cesàro or Hölder, except in the case of orders 0 and 1 . On the other hand, if $f_{r}(x)$ corresponds to $C_{r}$, the transformation of Cesàro of order $r$, according to the plan of type $A$, then the transformation $B_{r}$, obtained from $f_{r}(x)$ according to the plan of type $B$, is proved to be more general than $C_{r}$, for each positive integer $r$ greater than unity. The transformations of type $B$ may be written in the form of an integral, which is identical with the formula for the transformations of functions of a continuous variable.

37. Professor H. B. Phillips: Nets and the Dirichlet problem.

The author considers the function defined on a rectangular net by the condition that its value at each node is equal to the average of its values at the adjacent nodes. By considering nets with progressively finer mesh he approximates to the solution of the Dirichlet problem in the plane.

38. Professor H. C. M. Morse: When does the inverse problem of the calculus of variations lead to an integral of arc length?

The general inverse problem of the calculus of variations is to find an integral for which a given two-parameter family of curves are the extremals in case the integral be minimized. Darboux has shown that the general problem has an infinite number of solutions. The present writer inquires what are 
the conditions on the given family of curves that they may serve as extremals arising from an integral of arc length.

39. Mr. Louis Weisner: Groups whose maximal cyclic subgroups are independent.

A cyclic subgroup of a group is said to be a maximal cyclic subgroup of the group if it is not contained in any larger cyclic subgroup of the group. The present paper, which is the author's dissertation, concerns itself with groups whose maximal subgroups are independent; that is, groups in which no two maximal cyclic subgroups have an element in common besides identity. The cyclic group and the type $(1,1,1, \cdots)$ abelian groups are the only abelian groups having this property. Of prime power groups, the only ones are the cyclic, dihedral, the type $(1,1,1, \cdots)$ abelian group, and groups conformal with the type $(1,1,1, \cdots)$ abelian group. The only other solvable groups having the property are those which contain an invariant cyclic subgroup, and the octahedral group, which falls into a class by itself. All other such groups are insolvable and usually simple. General properties of such groups are also considered in the paper.

40. Dr. George Rutledge: The polynomial determined by $2 n+1$ evenly spaced points.

Examples of the polynomials whose general form the author discusses are the following: $\left(4-3 x^{2}\right) / 4,\left(52-51 x^{2}\right.$ $\left.+36 x^{4}\right) / 52, \quad\left(3,472-3,468 x^{2}+3,321 x^{4}-2,187 x^{6}\right) / 3,472$, $\left(48,412-48,409 x^{2}+48,204 x^{4}-44,928 x^{6}+27,648 x^{8}\right) / 48,412$, which agree in value with the function $1 /\left(1+x^{2}\right)=1-x^{2}$ $+x^{4}-x^{6}+\cdots$ for (respectively) three, five, seven, and nine equally spaced values of $x$ on the interval $x=-1 / \sqrt{3}$ to $x=+1 / \sqrt{3}$, end values included. These polynomials are the basis of many well known interpolation formulas.

41. Dr. E. L. Post: Visual intuition in spherical and elliptic space: Einstein's finite universe.

The method of the present paper is almost the same as the one used in the author's paper on Lobachevskian intuition. However, the difficulties involved in the conception of the finiteness of both the spherical and elliptic spaces and the one-sidedness of the latter require further elucidation. This is accomplished by imagining a plenitude of the straight lines of these spaces, uniformly distributed in them, and noting just how they bind the spaces together; or by noting how the spaces 
can be broken up into a finite number of adjacent but nonoverlapping cells. In both cases the desired intuition is obtained by noting how these straight lines and cells vary with changes in the position of the observer. Since Einstein's finite universe is one of these types, the present paper may serve to dispel some popular misconceptions.

42. Dr. E. L. Post: A non-Weierstrassian method of analytic prolongation.

The present method arose from an attempt to find satisfactory necessary and sufficient conditions that a function of a real variable be an analytic function. It was found that this was so when and only when a certain straight line construction which makes a network of broken lines correspond to a subdivision of a certain portion of the plane into squares gives in the limit a network of curves through whose parameterization a transformation of the plane is defined whose corresponding functions have continuous total differentials. Since at the same time the method gives the values of the original function over a region of the complex plane, whereas originally it was known only along a segment of the real axis, it appears as a new method of analytic prolongation. By repeated application of the method, the function can be explored throughout its domain of existence. The method is that used by R. G. D. Richardson in 1917. In its particular development it yields a neat formula for the coordinates of an arbitrary point of intersection of the network as a finite series whose terms approach those of a Taylor's expansion.

43. Dr. E. L. Post: A new method for generalizing $e^{x}$ in the complex domain.

The method in question is simply the one presented in the preceding paper. In the present case, the broken line network in the plane of the dependent variable becomes a set of lines radiating from the origin crossed orthogonally by a spider's web of broken lines. The lengths cut off along these radiating lines are found to be proportional to their distances from the origin, a fact which easily enables us to find that, in the limit, the network of broken lines is replaced by a pencil of lines crossed orthogonally by a series of concentric circles.

44. Dr. E. L. Post: A simple geometric proof of the equality of the Brochardt angles of a triangle.

It is easy to obtain a simple trigonometric proof of the equality of the Brochardt angles of a triangle, but as far as 
the author is aware no simple geometric proof of this fact is extant. The present note supplies such a proof.

45. Professor I. J. Schwatt: Method for the summation of series in which groups of positive terms alternate with groups of negative terms.

The author considers certain series in which the groups of positive terms and the groups of negative terms alternate and contain $m$ terms each. He represents these series as a sum of a finite number of integrals $I_{k}$, and he has obtained a formula which enables him to find the value of $I_{k}$ and similar integrals without going through the cumbersome work of integration.

46. Mr. Hillel Poritsky: On curves kinematically related to a given curve.

This paper appeared in the February Bulletin.

47. Professor F. L. Hitchcock: A method for the numerical solution of integral equations.

The purpose of this paper is to obtain a method requiring as little as possible in the way of evaluation of definite integrals. The Fredholm equation is treated over the interval from 0 to 1. It is shown that the only necessary definite integrals are (1) the integral of the given function $s(x)$ over this interval or over any desired finite part of this interval, and (2) the integral of the kernel $K(x, y)$ over a unit square or over any desired finite square contained within the unit square. The solution is obtained by a definite rule and the computation is suitable for carrying out with a calculating machine.

48. Professor Elizabeth B. Cowley: Further generalizations of the old mathematical puzzle of three vessels with capacities of 8,5 and 3.

This is a further development along the lines of the paper presented at the October meeting. Here the problem of four vessels is studied, starting with the cases where $A=B+C$ $+D$. It is found that, although certain cases can be solved by repeated use of one or both of the two methods employed for the three-vessel problem, it is necessary to devise other methods to meet the situation in cases where the three-vessel methods are not applicable. Some of these new methods can also be used in cases where the three-vessel methods are applicable. The conditions for the success or failure of the various methods are found. Certain modifications are necessary in cases where $A \gtrless B+C+D$. 
49. Mr. F. H. Murray: Real solutions of certain linear differential equations of the second order.

This paper is given to a study of the real bounded solutions of certain linear differential equations of a type discussed by Poincaré, Bohl, and Wiman. Besides a synthesis of some of the results obtained by these authors, certain new results are obtained by methods of successive approximations. In particular, an analytic expression for the general integral is obtained, from which many of the characteristic properties of the solutions follow immediately. A generalization of some of the results obtained by Bohl for linear equations with quasiperiodic coefficients is also given.

50. Mr. F. H. Murray: A type of arc-describing instrument. In this paper is described a type of instrument* for drawing arcs of circles of very large radius.

51. Mr. F. H. Murray: Certain isosceles triangle solutions in the problem of three bodies.

The aim of this paper is to deduce a certain inequality, satisfied by the isosceles triangle solutions with axis of symmetry, and non-coplanar, in the problem of three bodies, for large negative values of the energy constant. From this inequality and another found by J. Chazy, one can show that the solutions under consideration possess "complete stability," in the sense of Poincaré (Les Méthodes Nouvelles, III).

52. Professor J. W. Alexander: Invariant points of surface transformations.

Let $A_{1}, A_{2}, \cdots, A_{p}, B_{1}, B_{2}, \cdots, B_{p}$ be a canonical system of cuts on a closed surface of genus $p$, and let $\Delta$ be a transformation of the surface into itself carrying the cuts $A_{i}$ and $B_{i}$ into the cuts $\bar{A}_{i}$ and $\bar{B}_{i}$ respectively. Then

$$
\sum_{j} \tau_{j}=1+\tau+\sum_{i}\left\{N\left(B_{i} \bar{A}_{i}\right)+N\left(\bar{B}_{i} A_{i}\right)\right\},
$$

where $\Sigma \tau_{j}$ denotes the sum of the indices of the fixed points of the transformation, $\tau$ the index of the transformation itself, and $N(A B)$ the intersection number of the curves $A$ and $B$.

53. Mr. G. E. Raynor: On Dirichlet's problem for irregular boundaries.

The author gives an elementary solution of Dirichlet's problem under less restricted boundary conditions than those generally assumed.

R. G. D. Richardson, Secretary of the Society.

* These instruments are fully described in Letters Patent of the United States, No. 1,427,493, dated Aug. 29, 1922. 